2015

\title{
Prevention of ion flux lateral inhomogeneities in large area capacitive radio frequency plasmas via the electrical asymmetry effect
}

Edmund Schüngel

Sebastian Mohr

Julian Schulze

Uwe Czarnetzki

Follow this and additional works at: https://researchrepository.wvu.edu/faculty_publications

\section{Digital Commons Citation}

Schüngel, Edmund; Mohr, Sebastian; Schulze, Julian; and Czarnetzki, Uwe, "Prevention of ion flux lateral inhomogeneities in large area capacitive radio frequency plasmas via the electrical asymmetry effect" (2015). Faculty Scholarship. 292.

https://researchrepository.wvu.edu/faculty_publications/292 


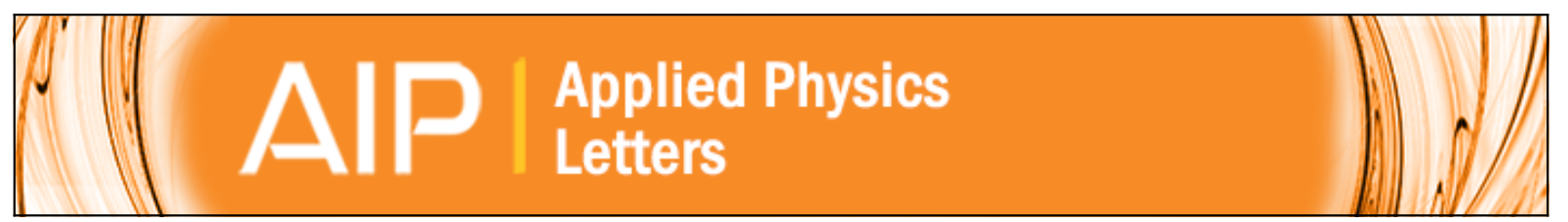

\section{Prevention of ion flux lateral inhomogeneities in large area capacitive radio frequency plasmas via the electrical asymmetry effect}

Edmund Schüngel, Sebastian Mohr, Julian Schulze, and Uwe Czarnetzki

Citation: Applied Physics Letters 106, 054108 (2015); doi: 10.1063/1.4907887

View online: http://dx.doi.org/10.1063/1.4907887

View Table of Contents: http://scitation.aip.org/content/aip/journal/apl/106/5?ver=pdfcov

Published by the AIP Publishing

\section{Articles you may be interested in}

Frequency dependence of the electrical asymmetry effect in dual-frequency capacitively coupled discharges Appl. Phys. Lett. 102, 154104 (2013); 10.1063/1.4802241

The electrical asymmetry effect in geometrically asymmetric capacitive radio frequency plasmas J. Appl. Phys. 112, 053302 (2012); 10.1063/1.4747914

Phase-shift effect in capacitively coupled plasmas with two radio frequency or very high frequency sources J. Appl. Phys. 108, 043308 (2010); 10.1063/1.3475376

Measurements and consequences of nonuniform radio frequency plasma potential due to surface asymmetry in large area radio frequency capacitive reactors

J. Vac. Sci. Technol. A 23, 922 (2005); 10.1116/1.1868572

Electric field distribution in plasma during ion extraction by a radio frequency resonance method Phys. Plasmas 4, 3527 (1997); 10.1063/1.872249

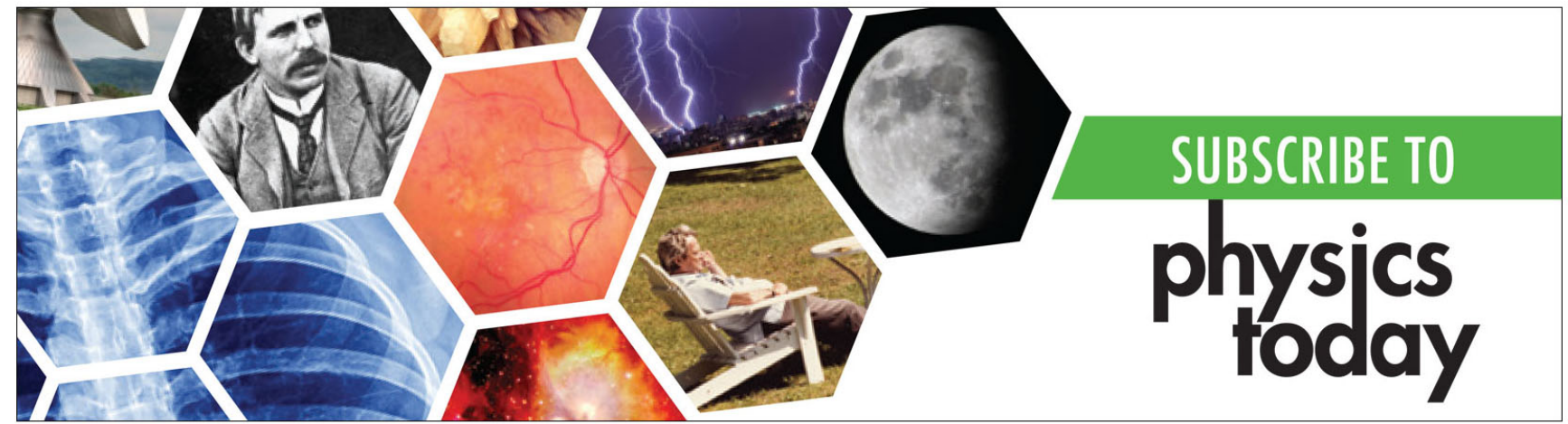




\title{
Prevention of ion flux lateral inhomogeneities in large area capacitive radio frequency plasmas via the electrical asymmetry effect
}

\author{
Edmund Schüngel, ${ }^{1}$ Sebastian Mohr, ${ }^{2}$ Julian Schulze, ${ }^{1}$ and Uwe Czarnetzki ${ }^{2}$ \\ ${ }^{1}$ Department of Physics, West Virginia University, Morgantown, West Virginia 26506-6315, USA \\ ${ }^{2}$ Institute for Plasma and Atomic Physics, Ruhr University Bochum, 44780 Bochum, Germany
}

(Received 3 December 2014; accepted 27 January 2015; published online 6 February 2015)

\begin{abstract}
The ion flux is measured spatially resolved along the substrate surface in dual-frequency large area capacitive discharges operated in $\mathrm{H}_{2}$ at $150 \mathrm{~Pa}$. One electrode is driven by $40.68 \mathrm{MHz}$ and/or 81.36 MHz. The dependence of the ion flux profile on the applied voltage waveform is investigated experimentally and by a two-dimensional model. The results show that lateral inhomogeneities caused by standing wave effects can be eliminated based on the electrical asymmetry effect, i.e., by tuning the phase shift between the two applied frequencies. This approach solves one of the major problems in plasma processing of large substrates. (C) 2015 AIP Publishing LLC.

[http://dx.doi.org/10.1063/1.4907887]
\end{abstract}

The manufacturing of many high technology products requires a plasma treatment of large surface areas. For instance, the plasma enhanced chemical vapor deposition (PECVD) of thin films is a crucial step in the production of polymorphous silicon solar cells or flat panel displays. Other applications range from computer chip manufacturing to the creation of biocompatible surfaces. Capacitively coupled radio frequency (CCRF) discharges are typically used for such processing applications, because they allow for the development of a chemically rich gas phase with controlled plasma surface reactions. ${ }^{1}$ Any improvements of process performance and efficiency typically result in enormous societal benefits and are, therefore, of general interest. For optimum process performance an excellent plasma uniformity at the substrate and a high process rate are required. The ion bombardment energy at the substrate must be controllable to avoid damages of the film growing on the substrate and, hence, optimize the film quality.

One way of increasing the deposition rate is the application of a higher excitation frequency to the powered electrode, thereby enhancing the plasma density ${ }^{2}$ and, subsequently, the deposition rate. ${ }^{3}$ However, this approach has major implications on the lateral homogeneity of the plasma properties. At high frequencies, the plasma-shortened ${ }^{1,4}$ wavelength corresponding to the applied frequency is on the same order as the electrode dimensions. Consequently, electromagnetic effects will significantly disturb the plasma uniformity. Depending on the specific discharge regime, standing wave and/or skin effects lead to ion flux inhomogeneities. Measurements, ${ }^{1,5,6}$ simulations, $^{7,8}$ and models ${ }^{1,4,6,9,10}$ have shown that the standing wave effect leads to a dome shaped ion flux profile with its maximum at the center of the electrodes, whereas the skin or the telegraph effect lead to ionization around the edges of the electrodes. Although advanced techniques such as the utilization of a lens shaped electrode, ${ }^{11,12}$ a segmentation of the powered electrode, ${ }^{13}$ and the use of material mixtures with radially graded conductivity ${ }^{14}$ have been developed, process and uniformity control in large area CCRF discharges operated at high driving frequencies remains a major challenge for both research and applications. The development of novel methods to control the ion flux profile, ideally based on a detailed understanding of the plasma kinetics, would allow for a more efficient surface processing, potentially resulting in multi-billion dollar savings in plasma processing applications.

Recently, a novel way to control the ion energy at the substrate separately from the ion flux by using the Electrical Asymmetry Effect (EAE) has been discovered. ${ }^{15-23}$ This separate control is realized by applying a voltage waveform, $\phi_{\sim}(t)$, consisting of two subsequent harmonics instead of only one frequency to the powered electrode

$$
\phi_{\sim}(t)=\phi_{1} \cos \left(\omega_{1} t+\theta_{1}\right)+\phi_{2} \cos \left(\omega_{2} t+\theta_{2}\right) .
$$

Here, $\omega_{1}=2 \pi f_{1}\left(f_{1}=40.68 \times 10^{6} \mathrm{~s}^{-1}\right), \omega_{2}=2 \omega_{1}, \phi_{1}, \phi_{2}$, $\theta_{1}$, and $\theta_{2}$ are the angular frequencies, voltage amplitudes, and phase shifts of the two Fourier components, respectively. The asymmetry of this applied voltage waveform, i.e., the difference between the absolute values of its global extremes, $\phi_{\sim}^{\max }$ and $\phi_{\sim}^{\min }$, depends on the relative phase $\theta_{\text {rel }}=\theta_{1}-\left(\theta_{2} / 2\right) \cdot{ }^{15}$ As a consequence, the symmetry of the entire CCRF discharge, the DC self-bias, $\eta$, and, consequently, the ion energy at the electrodes can be controlled via the EAE. ${ }^{15-19}$

Such voltage waveform tailoring has been demonstrated to be useful for the optimization of thin film depositions. ${ }^{24-26}$ However, all of these investigations have been restricted to electrostatic cases (using $13.56 \mathrm{MHz}$ and $27.12 \mathrm{MHz}$ ). Here, the EAE is examined in the electromagnetic regime, i.e., in a large area CCRF discharge operated at high frequencies for the first time. We study the effect of controlling the phase between the driving harmonics on the uniformity of the lateral ion flux profile at the substrate and demonstrate that the EAE can be used to strongly improve the plasma uniformity.

The basic experimental setup has been described in detail elsewhere. ${ }^{16,17}$ In brief, a geometrically symmetric parallel plate discharge is sustained by applying either a single-frequency (Eq. (1) with $\phi_{1}=0$ or $\phi_{2}=0$ ) or a dualfrequency (Eq. (1)) voltage waveform to the powered electrode, which exhibits a square surface area of $40 \times 40 \mathrm{~cm}^{2}$ and serves as a showerhead. The gap between the electrodes 

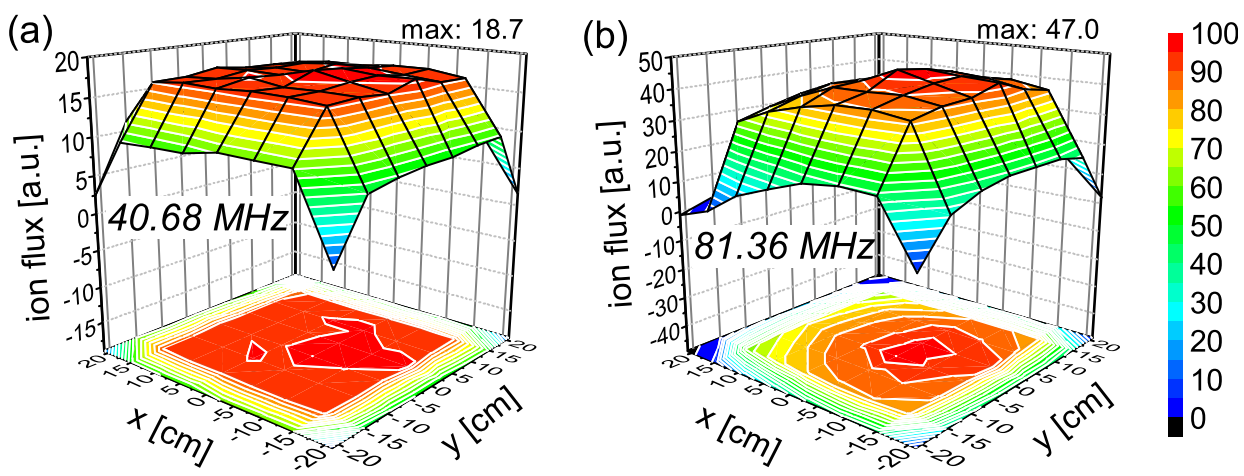

FIG. 1. Spatially resolved ion flux onto the grounded electrode measured in capacitive hydrogen discharges operated at $150 \mathrm{~Pa}$ and different applied radio frequencies. (a) $200 \mathrm{~W}$ at $40.68 \mathrm{MHz}$; (b) $450 \mathrm{~W}$ at $81.36 \mathrm{MHz}$. The color scale indicates percentages of the maximum flux of each profile, which is displayed at the right upper corner (in a.u.).

is $14 \mathrm{~mm}$. Accordingly, the pressure regime of stable discharge operation is relatively high and all dynamics in the plasma volume is strongly collisional. The plasma is laterally confined by a square grounded frame with an inner edge length of about $50 \mathrm{~cm}$. In order to measure the spatial profile of the ion flux to the grounded electrode, an array of $8 \times 8$ current sensors is implemented into this electrode. A bias voltage of $-9 \mathrm{~V}$ is applied to the circular sensors of $5 \mathrm{~mm}$ diameter. The driving frequencies are filtered from the signal, which is amplified and sequentially read out.

Before discussing the effect of an electrical asymmetry on the uniformity, the reference case of single frequency discharges shall be examined. Figure 1 shows the experimentally determined ion flux onto the grounded electrode as a function of position along the electrode surface for different driving frequencies. The center of the electrodes is also the center of the coordinate system. At a relatively low frequency of $40.68 \mathrm{MHz}$, the ion flux reveals a plateau shape. This is due to the fact that the plasma remains laterally uniform between the two electrodes, whereas the plasma density and, subsequently, the ion flux drops at the outer regions. It should be noted that the outmost current sensors are positioned at a lateral distance of about $1 \mathrm{~cm}$ outside of the edges of the powered electrode. In qualitative agreement with the measurements of Perret et al., ${ }^{5}$ the ion flux profile becomes dome shaped at the highest frequency investigated here $(81.36 \mathrm{MHz})$. This is a typical consequence of standing wave effects, as the voltage drop between the electrodes, i.e., across the two sheaths (and the plasma bulk), is maximum in the center and gradually decreases in all lateral directions. ${ }^{4-7,9,11}$ Furthermore, the maximum and the spatially averaged ion flux are more than two times larger in the 81.36 MHz discharge compared to the $40.68 \mathrm{MHz}$ case. This is due to the fact that a higher driving frequency leads to a more efficient electron heating, a higher current and plasma density, and higher fluxes. ${ }^{2}$ Although voltage measurements have not been possible at these high frequencies, we roughly estimate the voltage amplitude at both frequencies to be around $100 \mathrm{~V}$.

Figure 2 shows the ion flux profile of a capacitive discharge using the EAE at high frequencies, i.e., applying 40.68 MHz and $81.36 \mathrm{MHz}$, for different values of the relative phase shift and, hence, different values of the DC selfbias, $\eta$. It can be inferred from the experimentally obtained DC self-bias and previous results at lower frequencies ${ }^{15-17,20,21}$ that the relative phase of the driving harmonics is approximately $\theta_{\text {rel }} \approx 0^{\circ}, 45^{\circ}$, and $90^{\circ}$ in Figures 2(a), 2(b), and 2(c), respectively. Here, we estimate a spatially averaged phase; as will be shown below, $\theta_{\text {rel }}$ varies laterally. For small phase angles and negative DC self-bias values, the spatially resolved ion flux profile resembles an intermediate case between the profiles measured in single frequency discharges operated at $40.68 \mathrm{MHz}$ and $81.36 \mathrm{MHz}$ (Figures 1(a) and 1(b)). However, tuning the phase angle to values around $90^{\circ}$ and, accordingly, the DC self-bias to maximum positive values, changes the lateral distribution of the ion flux completely. Instead of a dome shaped profile, the ions flow almost equally to all positions covered by the current sensors. In contrast to all other cases including the operation of the CCRF discharge using lower frequencies, there is a significant ion flux at the outmost positions. Defining the region of uniformity as all areas, where the ion flux deviates by not more than $10 \%$ from the peak value, we find that 36 of 64 probes or about $56.3 \%$ fall within this criterion at a low driving frequency of $40.68 \mathrm{MHz}$ (Figure 1(a)). At a high driving frequency, the standing wave effect reduces the region of uniformity to $15.6 \%$ (Figure 1(b)). Using the EAE at $40.68 \mathrm{MHz}$ and $81.36 \mathrm{MHz}$ (Figure 2(c)), however, about $73.4 \%$ of the probed surface area stays within the limit of $10 \%$ non-uniformity. Further, it is important to note that the peak and the total ion flux are even higher compared to the single high-frequency $(81.36 \mathrm{MHz})$ case. Altogether, the very high and uniform flux facilitated by controlling the phase between two high applied frequencies would mean a substantial benefit in terms of high and uniform process rates in applications.

One important physical mechanism, which might cause the dependence of the ion flux profile on the relative phase angle, is that the voltage across the grounded sidewall sheath and, thereby, the excitation strength of the telegraph effect $^{6,10}$ is varied via the EAE by tuning the relative phase, $\theta_{\text {rel. }}{ }^{15-18}$ In particular, this sidewall sheath is largest around $\theta_{\text {rel }} \approx 90^{\circ}$ so that the power dissipated to electrons in the outer region is enhanced. In fact, the situation is even more complicated, as this phase must exhibit a dependence on the lateral position along the electrode surface. This is a result of the dependence of the damping and phase shifting of waves propagating through the plasma on the wave frequency, i.e., of the plasma dispersion relation, and has multiple consequences on the plasma properties and dynamics.

The effects of a radial variation of the relative phase can be illustrated, for instance, in the frame of a simplified two- 


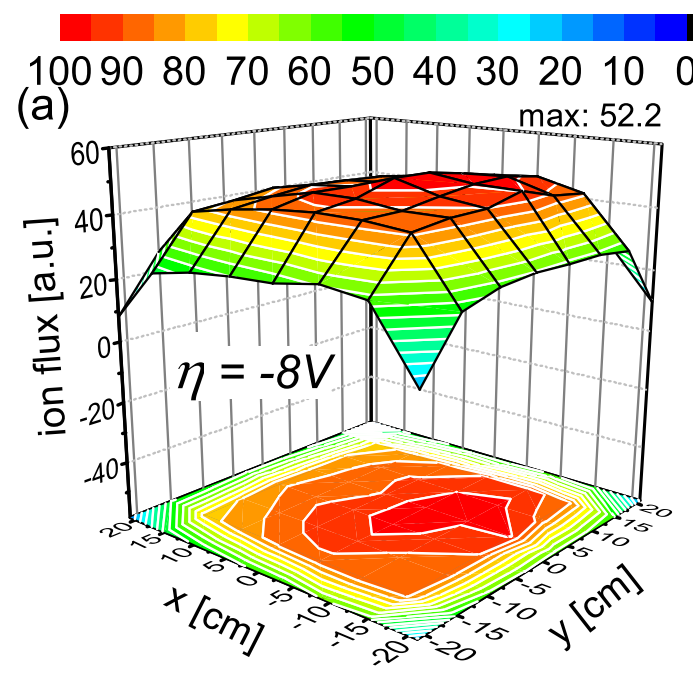

(b) $\quad \max : 48.2$

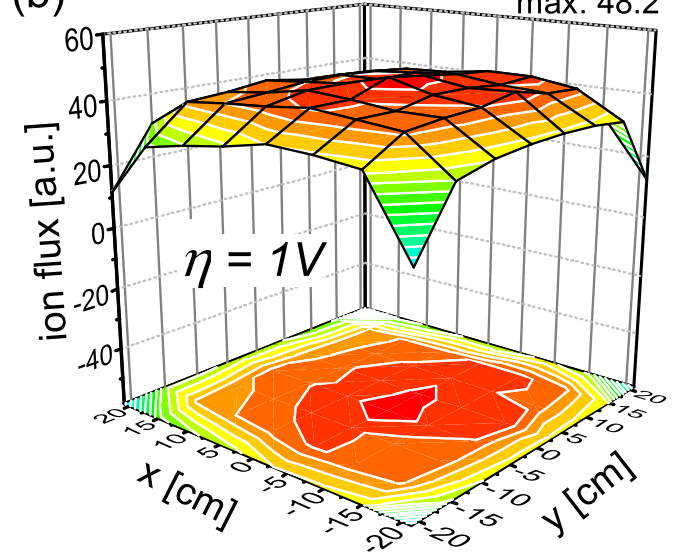

(c) $\quad \max : 57.0$

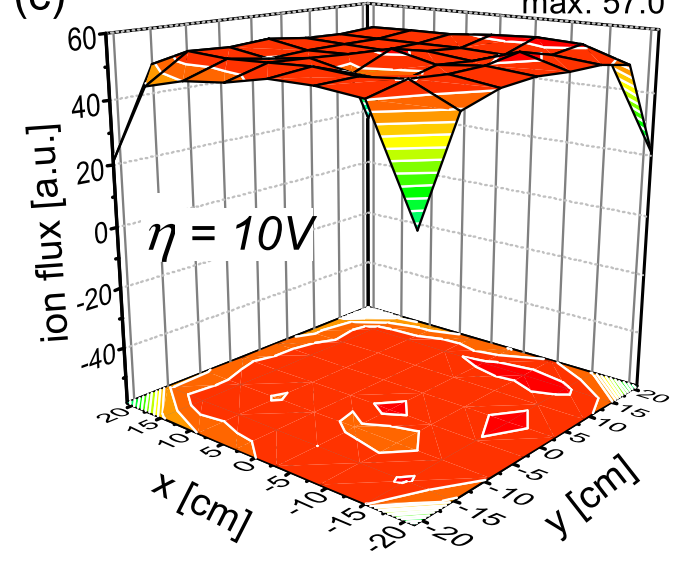

FIG. 2. Spatially resolved ion flux onto the grounded electrode measured in electrically asymmetric capacitive hydrogen discharges operated at a combination of $40.68 \mathrm{MHz}$ and $81.36 \mathrm{MHz}$. (a) minimum negative DC self-bias; (b) intermediate DC self-bias; (c) maximum positive DC self-bias. The applied power is kept constant at $200 \mathrm{~W}(40.68 \mathrm{MHz})$ and $450 \mathrm{~W}$ $(81.36 \mathrm{MHz})$. The color scale indicates percentages of the maximum flux of each profile, which is displayed at the right upper corner (in a.u.).

dimensional model, which has been introduced by Sansonnens et al. in Ref. 10. As shown in Figure 3, a cylindrical CCRF discharge is treated as a homogeneous plasma slab of thickness $l_{b}$ and radius $R$, which is separated from the surrounding surfaces by vacuum sheaths of thickness $s$. The plasma is treated as a dispersive medium, so that each Fourier component is considered individually. We use the expressions

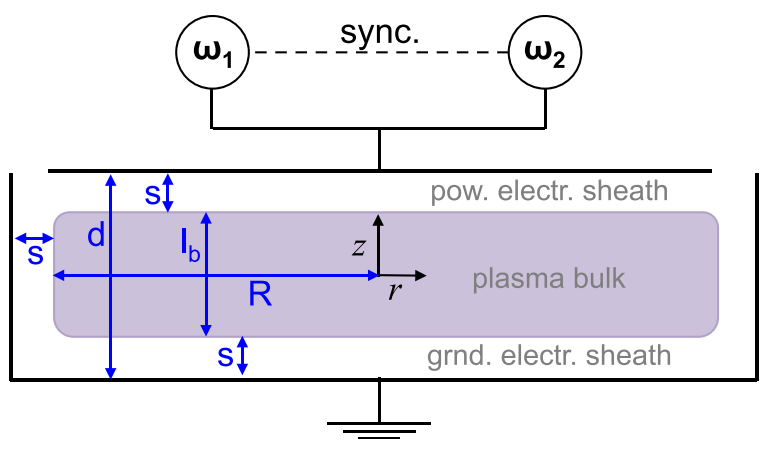

FIG. 3. Illustration of the cylindrical geometry of the two-dimensional model, showing the coordinate system and the relevant spatial quantities.

(8)-(16) in the original work ${ }^{10}$ to calculate the electromagnetic field in the bulk and in the two sheaths, respectively. The complex wavenumbers of the propagation of electromagnetic waves in the radial and axial directions are obtained by the dispersion relations and the continuity of the fields at the plasma sheath transitions, i.e., at $z= \pm l_{b} / 2$ (Eqs. (6), (7), (17), and (18) in Ref. 10). Here, we exemplarily examine the case of $\quad d=14 \mathrm{~mm}, \quad s=3 \mathrm{~mm}, \quad l_{b}=8 \mathrm{~mm}, \quad R=\sqrt{(0.4 \mathrm{~m})^{2} / \pi}$ $\approx 0.226 \mathrm{~m}, \omega_{p e}=20 \omega_{1}\left(\omega_{1}=2 \pi 40.68 \times 10^{6} \mathrm{~s}^{-1}\right.$, equivalent to an electron density of $\left.8.2 \times 10^{15} \mathrm{~m}^{-3}\right)$, and $\nu_{m}=10 \omega_{1}{ }^{27,28}$ The amplitudes are normalized at the center, i.e., $\phi_{1}(r=0)=\phi_{2}(r=0)=1$. The interelectrode voltage is obtained via Poisson's equation, $\phi_{\sim}^{E M}(r)=-\int E(r, z) d z$. Naturally, the resulting voltage will be a dual-frequency waveform, whereas the amplitude and phase of each Fourier component changes along the electrodes.

Based on this simple model, we find that the relative phase decreases by about $15.5^{\circ}$ between the discharge center $(r=0)$ and the outer region $(r=0.9 R)$. The main reason is the superposition of the odd mode (telegraph effect) decaying along the propagation from the sidewall sheath towards the discharge center with the dome shaped profile of the even mode (standing wave effect). This leads to a significant difference of the voltage waveform at different radii (see Figure 4). As a consequence thereof, the maximum and minimum of the local voltage drop across the discharge and, thus, the electrical asymmetry vary along the radial propagation

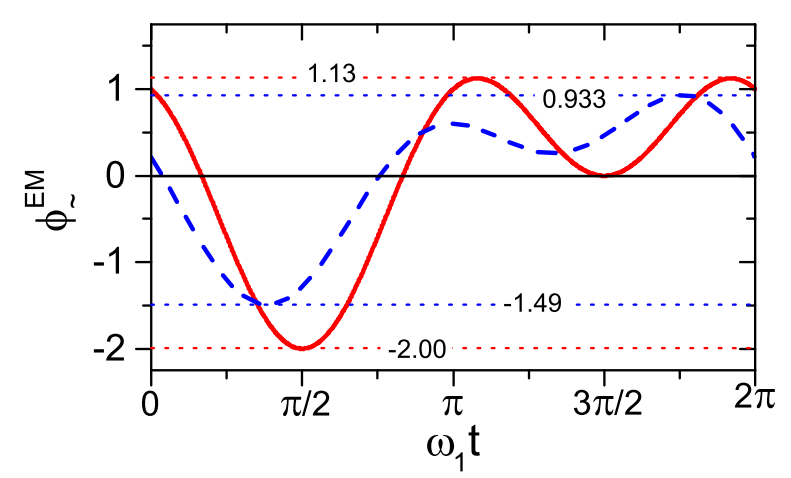

FIG. 4. RF interelectrode voltage drop at the center $(r=0$, red solid line) and at the outer region $(r=0.9 R$, blue dashed line). The phase shifts are set to $\theta_{1}^{E M}=90^{\circ}$ and $\theta_{2}^{E M}=0^{\circ}$ and the amplitudes of both frequency components are normalized at the center. The dotted lines indicate extreme values. 
path. This must not correspond to a change of the DC selfbias, as it is a global quantity for perfectly conducting electrode surface areas. Therefore, the DC self-bias needs to be kept spatially constant by a lateral dependence of the maximum sheath voltages: Based on the model introduced by Heil et al., ${ }^{15}$ the DC self-bias, $\eta$, is linked to the global extremes, $\phi_{\sim}^{\max }$ and $\phi_{\sim}^{\min }$, of the applied voltage waveform and the absolute value of the ratio of the maximum sheath voltages, i.e., the so called symmetry parameter, $\varepsilon:^{15,18}$

$$
\varepsilon(r)=\left|\frac{\phi_{s g}^{\max }(r)}{\phi_{s p}^{\max }(r)}\right|=-\frac{\eta+\phi_{\sim}^{\max }(r)}{\eta+\phi_{\sim}^{\min }(r)} .
$$

The symmetry parameter $\varepsilon$ (Eq. (2)) needs to vary laterally by at least about $30 \%$ in this model assuming $\varepsilon=1$ at $r=0$. It is larger at the outer region compared to the discharge center. Accordingly, the maximum powered electrode sheath voltage, $\phi_{s p}^{\max }$, decreases and/or the maximum voltage drop across the grounded electrode sheath, $\phi_{s g}^{\max }$, increases from the center to the edges. This will enhance the electron heating dynamics and, eventually, the ion flux onto the grounded electrode in the outer region.

A spatial variation of $\theta_{r e l}$ affects the electron heating dynamics. Since the electron heating in hydrogen discharges is dominated by the rapid expansion of each of the sheaths and the field reversals around the times of sheath collapse, ${ }^{20,29-32}$ the temporal behavior, total heating, and subsequent ionization strongly depend on the specific (local) voltage waveform. In particular, the decrease of the voltage at $\omega_{1} t \approx \pi$ observed in Figure 4 reduces the number of total sheath collapses, which alters the electron heating by electric field reversals.

Certainly, radial variations of the plasma asymmetry or more complicated effects such as the coupling between the two harmonics due to the sheaths nonlinearities ${ }^{33}$ cannot be captured self-consistently in this model. Therefore, while the above description provides a general idea of how the ion flux is kept uniform, further studies (e.g., using self-consistent, kinetic, and electromagnetic simulations ${ }^{7}$ ) are required to ultimately explain these important experimental findings.

In conclusion, the experimental investigation of the ion flux in the electromagnetic regime of CCRF discharges revealed that ion flux inhomogeneities due to standing wave effects can be eliminated by choosing an electrically asymmetric applied voltage waveform with a specific phase shift between the applied frequencies. Furthermore, the homogeneity of the ion flux, which is a crucial quantity in large area surface processing applications of capacitive discharges, can be significantly improved compared to the standard (single-frequency) case. The homogeneity control works without any modifications of the plasma chamber and can be easily adapted to any RF plasma process. A hypothetical description of the underlying physics is presented in terms of a simplified model, which shows that the experimental findings can be consistently explained by the telegraph effect. ${ }^{6,10}$ The general insights into the lateral control of the electron heating dynamics by local voltage waveform effects can be extended to other gases.

Funding by the German Federal Ministry for the Environment, Nature conservation, and Nuclear Safety (0325210B) is gratefully acknowledged.

${ }^{1}$ P. Chabert and N. Braithwaite, Physics of Radio-Frequency Plasmas (Cambridge University Press, Cambridge, 2001).

${ }^{2}$ V. Vahedi, C. K. Birdsall, M. A. Lieberman, G. DiPeso, and T. D. Rognlien, Phys. Fluids B 5, 2719 (1993).

${ }^{3}$ U. Kroll, A. Shah, H. Keppner, J. Meier, P. Torres, and D. Fischer, Sol. Energy Mater. Solar Cells 48, 343 (1997).

${ }^{4}$ P. Chabert, J. L. Raimbault, J. M. Rax, and M. A. Lieberman, Phys. Plasmas 11, 1775 (2004).

${ }^{5}$ A. Perret, P. Chabert, J.-P. Booth, J. Jolly, J. Guillon, and Ph. Auvray, Appl. Phys. Lett. 83, 243 (2003).

${ }^{6}$ A. Howling, L. Derendinger, L. Sansonnens, H. Schmidt, C. Hollenstein, E. Sakanaka, and J. Schmitt, J. Appl. Phys. 97, 123308 (2005).

${ }^{7}$ D. Eremin, T. Hemke, R. P. Brinkmann, and T. Mussenbrock, J. Phys. D: Appl. Phys. 46, 084017 (2013).

${ }^{8}$ Y.-R. Zhang, X. Xu, A. Bogaerts, and Y.-N. Wang, J. Phys. D: Appl. Phys. 45, 015202 (2012).

${ }^{9}$ M. A. Lieberman, J. P. Booth, P. Chabert, J. M. Rax, and M. M. Turner, Plasma Sources Sci. Technol. 11, 283 (2002).

${ }^{10}$ L. Sansonnens, A. A. Howling, and Ch. Hollenstein, Plasma Sources Sci. Technol. 15, 302 (2006).

${ }^{11}$ H. Schmidt, L. Sansonnens, A. A. Howling, Ch. Hollenstein, M. Elyaakoubi, and J. P. M. Schmitt, J. Appl. Phys. 95, 4559 (2004).

${ }^{12}$ L. Sansonnens and J. Schmitt, Appl. Phys. Lett. 82, 182 (2003).

${ }^{13}$ Y. Yang and M. J. Kushner, J. Phys. D: Appl. Phys. 43, 152001 (2010).

${ }^{14}$ Y. Yang and M. J. Kushner, J. Appl. Phys. 108, 113306 (2010).

${ }^{15}$ B. G. Heil, U. Czarnetzki, R. P. Brinkmann, and T. Mussenbrock, J. Phys. D: Appl. Phys. 41, 165202 (2008).

${ }^{16}$ E. Schüngel, S. Mohr, S. Iwashita, J. Schulze, and U. Czarnetzki, J. Phys. D: Appl. Phys. 46, 175205 (2013).

${ }^{17}$ E. Schüngel, Ph.D. dissertation, Ruhr-University Bochum, 2013.

${ }^{18}$ E. Schüngel, D. Eremin, J. Schulze, T. Mussenbrock, and U. Czarnetzki, J. Appl. Phys. 112, 053302 (2012).

${ }^{19}$ T. Lafleur, P. A. Delattre, E. V. Johnson, and J. P. Booth, Appl. Phys. Lett. 101, 124104 (2012).

${ }^{20}$ S. Mohr, E. Schüngel, J. Schulze, and U. Czarnetzki, J. Phys. D: Appl. Phys. 46, 435201 (2013).

${ }^{21}$ S. Bienholz, T. Styrnoll, and P. Awakowicz, J. Phys. D: Appl. Phys. 47, 065201 (2014).

${ }^{22}$ E. Schüngel, J. Schulze, Z. Donkó, and U. Czarnetzki, Phys. Plasmas 18, 013503 (2011).

${ }^{23}$ T. Lafleur, P. Chabert, M. M. Turner, and J. P. Booth, Plasma Sources Sci. Technol. 22, 065013 (2013).

${ }^{24}$ E. V. Johnson, T. Verbeke, J.-C. Vanel, and J.-P. Booth, J. Phys. D: Appl. Phys. 43, 412001 (2010).

${ }^{25}$ E. V. Johnson, S. Pouliquen, P. A. Delattre, and J.-P. Booth, J. Non-Cryst. Solids 358, 1974 (2012).

${ }^{26}$ D. Hrunski, F. Mootz, A. Zeuner, A. Janssen, H. Rost, R. Beckmann, S. Binder, E. Schüngel, S. Mohr, D. Luggenhölscher, U. Czarnetzki, and G. Grabosch, Vacuum 87, 114 (2013).

${ }^{27}$ A. V. Phelps, Compilation of atomic and molecular data available at http://jilawww.colorado.edu/ avp/collision_data.

${ }^{28}$ A. V. Phelps, J. Phys. Chem. Ref. Data 19, 653 (1990).

${ }^{29}$ C. M. O. Mahony, R. Al Wazzan, and W. G. Graham, Appl. Phys. Lett. 71, 608 (1997).

${ }^{30}$ F. Tochikubo, T. Makabe, S. Kakuta, and A. Suzuki, J. Appl. Phys. 71, 2143 (1992).

${ }^{31}$ S. B. Radovanov, K. Dzierzega, J. R. Roberts, and J. K. Olthoff, Appl. Phys. Lett. 66, 2637 (1995).

${ }^{32}$ U. Czarnetzki, D. Luggenhölscher, and H. F. Döbele, Plasma Sources Sci. Technol. 8, 230 (1999).

${ }^{33}$ J. Robiche, P. C. Boyle, M. M. Turner, and A. R. Ellingboe, J. Phys. D: Appl. Phys. 36, 1810 (2003). 